\title{
A Novel Self-Calibration Method for Acoustic Vector Sensor
}

\author{
Yao Zhang $\mathbb{D}^{1}{ }^{1}$ Jin $\mathrm{Fu}^{2}{ }^{2}$ and Guannan $\mathrm{Li}^{1}$ \\ ${ }^{1}$ State Key Laboratory of Robotics, Shenyang Institute of Automation, Chinese Academy of Sciences, Shenyang 110016, China \\ ${ }^{2}$ National Laboratory of Underwater Acoustic Technology, Harbin Engineering University, Harbin 150001, China \\ Correspondence should be addressed to Yao Zhang; zhangyao@sia.cn
}

Received 7 August 2017; Revised 16 January 2018; Accepted 31 January 2018; Published 11 March 2018

Academic Editor: Haranath Kar

Copyright (C) 2018 Yao Zhang et al. This is an open access article distributed under the Creative Commons Attribution License, which permits unrestricted use, distribution, and reproduction in any medium, provided the original work is properly cited.

\begin{abstract}
The acoustic vector sensor (AVS) can measure the acoustic pressure field's spatial gradient, so it has directionality. But its channels may have nonideal gain/phase responses, which will severely degrade its performance in finding source direction. To solve this problem, in this study, a self-calibration algorithm based on all-phase FFT spectrum analysis is proposed. This method is "selfcalibrated" because prior knowledge of the training signal's arrival angle is not required. By measuring signals from different directions, the initial phase can be achieved by taking the all-phase FFT transform to each channel. We use the amplitude of the main spectrum peak of every channel in different direction to formulate an equation; the amplitude gain estimates can be achieved by solving this equation. In order to get better estimation accuracy, bearing difference of different training signals should be larger than a threshold, which is related to SNR. Finally, the reference signal's direction of arrival can be estimated. This method is easy to implement and has advantage in accuracy and antinoise. The efficacy of this proposed scheme is verified with simulation results.
\end{abstract}

\section{Introduction}

Different from the scalar sensor which can only measure the sound pressure signal, the AVS (acoustic vector sensor, also as vector hydrophone) consists of three identical, but orthogonally oriented, acoustic velocity sensors, all spatially colocated in a point-like geometry. Thus the AVS treats the acoustic wavefield as a vector field, then it can measure the sound pressure and particle velocity (or acceleration) of one point. The AVS has many advantages, such as frequency-independent dipole directivity, being good at suppressing isotropic noise, and estimating the direction without left-right ambiguity. These features can provide new ways to solve many underwater acoustic problems. And the performance of the underwater acoustic measurement system can be improved. With the development of the technology of sensor making and signal processing, the AVS has been widely used in military and civil application [1-10].

Because of the difference in production process and amplification, each channel's amplitude gain and phase of the AVS may have uncertainty. Besides, the changing of application environment may also change the parameters of the AVS. So the accuracy of the DOA (directions-of-arrival) estimation methods may decrease severely. As a result, the AVS needs to be calibrated at different frequency and azimuth before use.

A few calibration methods are devised for AVS's misorientation. The well-known calibration method is the comparison method. This method uses a standard hydrophone as a benchmark to adjusting the angles of the source and handles AVS's misorientation by performing aided calibration, necessitating cooperative emitters to impinge from prior known DOAs. Another calibration method is the vibration liquid column method. This method does not need a standard hydrophone as a reference. The AVS can be calibrated with an accelerometer and relative instrument. It means the above method needs special equipment and sealed cabin (or anechoic tank), making it inconvenient and high in cost. A typical calibration tank is shown in Figure 1. Some "selfcalibration" methods (also called "blind" or "quasiblind" methods that calibrate without prior information of the DOA of the calibrating source) are proposed. These methods are usually simple, easy to operate, and real-time, but most of them focus on the array of sensors [11-16]. Reference [17] calibrates the misorientation (not gain/phase uncertainty) of AVS, but it requires prior knowledge of the AVS's perfect orientation at a prior moment. Reference [18] only calibrates 


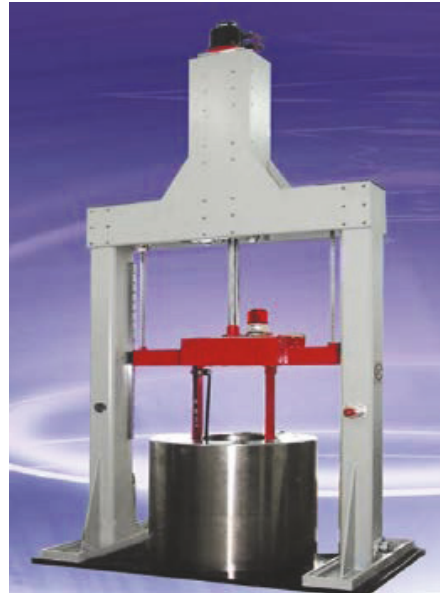

FIGURE 1: Typical calibration tank.

the intervector-sensors' misorientation and requires an openended iteration.

In this paper, a self-calibration algorithm for single AVS is proposed. This method can fix the phase and amplitude uncertainties without knowing the DOAs of the calibrating source in advance. The initial phase and amplitude estimation of all channels are obtained with AP-FFT (all-phase fast Fourier transforms). Then one channel's phase is used as a reference to compensate other channels. The amplitude gain estimation of each channel is obtained using the measurement of different azimuths according to the relationship of each channel. By using the phase and amplitude estimation result, the high accuracy of DOA estimation can finally be achieved.

This paper is organised as follows: Section 2 introduces the measurement model of the AVS. The MUSIC (Multiple Signal Classification) algorithm on DOA estimation and the influence of amplitude and phase deviation on DOA estimation are discussed separately. Section 3 presents the formulation and derivation of the proposed self-calibration algorithm. The performance of the algorithm is then presented in Section 4. The work is concluded in Section 5.

\section{Algorithm Research for AVS}

2.1. Measurement Model of Acoustic Vector Sensor. Generally, an AVS is composed of a pressure hydrophone and three particle velocity hydrophones. The pressure sensor measures the sound pressure, and the particle velocity sensor can measure the particle vibration velocity of the sound field.

In a far field plane sound wave field, the sound propagates towards certain direction. The acoustic pressure $p(x, t)$ can be expressed as

$$
p(x, t)=p_{0} e^{j(\omega t-k x)} .
$$

In the above formula, $p_{0}$ is the amplitude, $\omega$ is the angular frequency, and the wavenumber $s=\omega / c=2 \pi / \lambda$. The pressure gradient can be expressed as

$$
\nabla p=\frac{d p(x, t)}{d x}=-j k p(x, t)=-j \frac{\omega}{c} p(x, t) .
$$

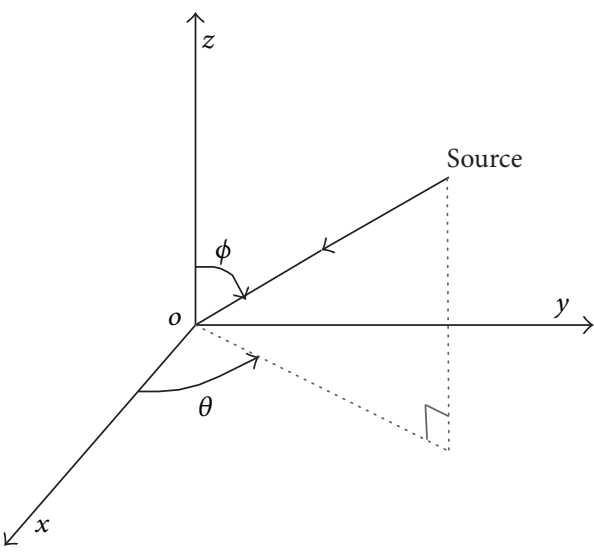

FIGURE 2: Vector sensor measurement model.

Based on Euler equation, the expression of the vibration velocity function is

$$
\begin{aligned}
v(x, t) & =-\frac{1}{\rho} \int \nabla p d t=\frac{1}{\rho} \int j k p_{0} e^{j(\omega t-k x)} d t \\
& =\frac{1}{\rho c} p_{0} e^{j(\omega t-k x)}=\frac{1}{\rho c} p(x, t),
\end{aligned}
$$

where $\rho$ represents the density of the medium and $\rho c$ is the acoustical wave impedance. The pressure of a plane wave can be expressed as the superposition of harmonic plane waves,

$$
\begin{aligned}
p(r, t) & =\int X(\omega) e^{j(\omega t-k r)} d \omega, \\
X(\omega) & =\int x(t) e^{-j \omega t} d t .
\end{aligned}
$$

$X(\omega)$ is the spectrum of $x(t)$; substituting formula (4) into formula (3), then

$$
\vec{v}(x, t)=-\frac{1}{\rho} \iint \nabla\left[X(\omega) e^{j(\omega t-k x)}\right] d \omega d t
$$

Take integral to $t$ :

$$
\begin{aligned}
& \vec{v}(x, t)=-\frac{1}{\rho} \int \nabla\left[\frac{X(\omega)}{j \omega} \cdot e^{j(\omega t-k x)}\right] d \omega \\
& =\frac{1}{\rho c}[\cos \theta \sin \phi \vec{\zeta}+\sin \theta \sin \phi \vec{\eta}+\cos \phi \vec{\xi}] p(x, t),
\end{aligned}
$$

where $\vec{\zeta}, \vec{\eta}, \vec{\xi}$ are unit vectors orthogonal to each other. If the influence of acoustical wave impedance can be ignored, the particle velocity is the only cosine weight of the sound pressure signal projected in three mutually orthogonal directions. They have the same waveform and the same or contrary phase and are completely related. The difference is the amplitude caused by the azimuth of the received signal.

The measurement model of AVS is shown in Figure 2. 
As $s(t)=\left[p(t), v_{x}(t), v_{y}(t), v_{z}(t)\right]^{T}$ is the observation data. The steering vector $A$ is written as

$$
A=\left[\begin{array}{l}
a_{p} \\
a_{x} \\
a_{y} \\
a_{z}
\end{array}\right]=\left[\begin{array}{c}
1 \\
\cos \theta \sin \phi \\
\sin \theta \sin \phi \\
\cos \phi
\end{array}\right] .
$$

The received signal $S(t)$ is

$$
\begin{aligned}
S(t) & =A x(t)+N(t) \\
& =\left[\begin{array}{c}
1 \\
\cos \theta \sin \phi \\
\sin \theta \sin \phi \\
\cos \phi
\end{array}\right] x(t)+\left[\begin{array}{l}
n_{p}(t) \\
n_{x}(t) \\
n_{y}(t) \\
n_{z}(t)
\end{array}\right],
\end{aligned}
$$

where $N(t)=\left[n_{p}(t), n_{x}(t), n_{y}(t), n_{z}(t)\right]^{T}$ is the ambient noise.

\subsection{Acoustic Vector Sensor Target Azimuth Estimation} Method. The main methods for a single AVS to estimates the DOA are as follows: the average sound intensity method, the cross spectrum method, and the multiple signal reconstruction method.

The average sound intensity method estimates the DOA by calculating the sound intensity of each channel. The average sound intensity is defined as

$$
I_{i}(t)=\overline{P(t) \cdot V_{i}(t)}, \quad i=x, y, z,
$$

where $\overline{()}$ means calculating the average. Then the DOA of received signal can be obtained with the following formula:

$$
\begin{aligned}
& \widehat{\theta}(t)=\arctan \left[\frac{I_{y}(t)}{I_{x}(t)}\right], \\
& \widehat{\phi}(t)=\arctan \left[\frac{\sqrt{I_{x}^{2}(t)+I_{y}^{2}(t)}}{I_{z}(t)}\right] .
\end{aligned}
$$

The cross spectrum method first calculates the Fourier transform of the received signal in each channel and then gets the DOA estimation by computing the complex sound intensity. Signal's complex sound intensity is defined as

$$
I_{i}(\omega)=P(\omega) \cdot V_{i}^{*}(\omega), \quad i=x, y, z,
$$

where $*$ represents calculating the conjugate operation. The real part of $I_{i}(\omega)$ is the active sound intensity. Then the azimuth of the source can be obtained as

$$
\begin{aligned}
& \hat{\theta}(\omega)=\arctan \left[\frac{\operatorname{Re}\left[I_{y}(\omega)\right]}{\operatorname{Re}\left[I_{x}(\omega)\right]}\right], \\
& \hat{\phi}(\omega)=\arctan \left[\frac{\sqrt{\left(\operatorname{Re}\left[I_{x}(\omega)\right]\right)^{2}+\left(\operatorname{Re}\left[I_{y}(\omega)\right]\right)^{2}}}{\left(\operatorname{Re}\left[I_{z}(\omega)\right]\right)^{2}}\right] .
\end{aligned}
$$

Since this method is calculated in frequency domain, the multisignals with different frequency can be resolved.

Schmidt's MUSIC (Multiple Signal Classification) algorithm is the most famous high-resolution subspace DOA estimation algorithm. Based on the MUSIC algorithm, typical subspace algorithms include feature eigenvector method, minimum-norm (min-norm) algorithm, and other improved algorithms.

Assuming the number of target $k$ is known $(k=1,2)$, the received ambient noise by each channel of the vector sensor is zero-mean white noise and uncorrelated. By calculating $M \times$ $M(M=3,4)$ dimensional covariance matrix, the eigenvalue decomposition can be written as

$$
\mathbf{R}=\mathbf{U}_{s} \Sigma_{s} \mathbf{U}_{s}^{H}+\mathbf{U}_{n} \Sigma_{n} \mathbf{U}_{n}^{H},
$$

where $\mathbf{U}_{s}$ is the Eigen subspace from the eigenvector and the eigenvector is corresponding to the $K$ eigenvalues, which is called the signal subspace. $\mathbf{U}_{n}$ is the eigensubspace from $M-K$ eigenvalues from the noise subspace. In ideal situation, the signal subspace is orthogonal to the noise subspace; thus the expression of the spatial spectrum of the MUSIC algorithm can be obtained as

$$
P_{\text {MUSIC }}(\theta)=\frac{1}{\mathbf{a}^{H}(\theta, \phi) \mathbf{U}_{n} \mathbf{U}_{n}^{H} \mathbf{a}(\theta, \phi)} .
$$

The spectral peak of the MUSIC algorithm can accurately show the DOA of the received signal. As long as the integration time is long enough, the signal-to-noise ratio (SNR) is high, the array model is accurate, and the azimuth estimation result can reach enough accuracy. However, due to the finite time of the received data and the influence of the noise, the signal subspace and the noise subspace are not completely orthogonal. So the minimum optimization search is needed to obtain the DOA.

\subsection{The Influence of Amplitude Phase Uncertainties on DOA} Estimation. In order to correctly show the pressure and particle velocity information of the sound field, the amplitude and phase characteristics of the AVS should be consistent. However, it is hard to keep them constant in practical applying due to the position changing, the gain difference of each channel, and other reasons. In this situation, the measurement model can be expressed as

$$
S(t)=\Gamma A X(t)+N(t),
$$

where $\Gamma=G \Phi$ is the gain/phase responses error matrix, $G=$ $\operatorname{diag}\left[g_{p}, g_{x}, g_{y}, g_{z}\right]$ is the amplitude gain coefficient vector, and $\Phi=\operatorname{diag}\left[e^{j \varphi p}, e^{j \varphi x}, e^{j \varphi y}, e^{j \varphi z}\right]$ is the phase error vector.

When using the MUSIC algorithm, formula (14) becomes

$$
P_{\text {MUSIC }}(\theta)=\frac{1}{(\Gamma A(\theta, \varphi))^{H} U_{n} U_{n}^{H}(\Gamma A(\theta, \varphi))} .
$$

The influence of nonideal gain/phase responses to the MUSIC algorithm is already analysed by many papers. The amplitude or phase error between the sound pressure and the velocity channel will not affect the azimuth estimation 


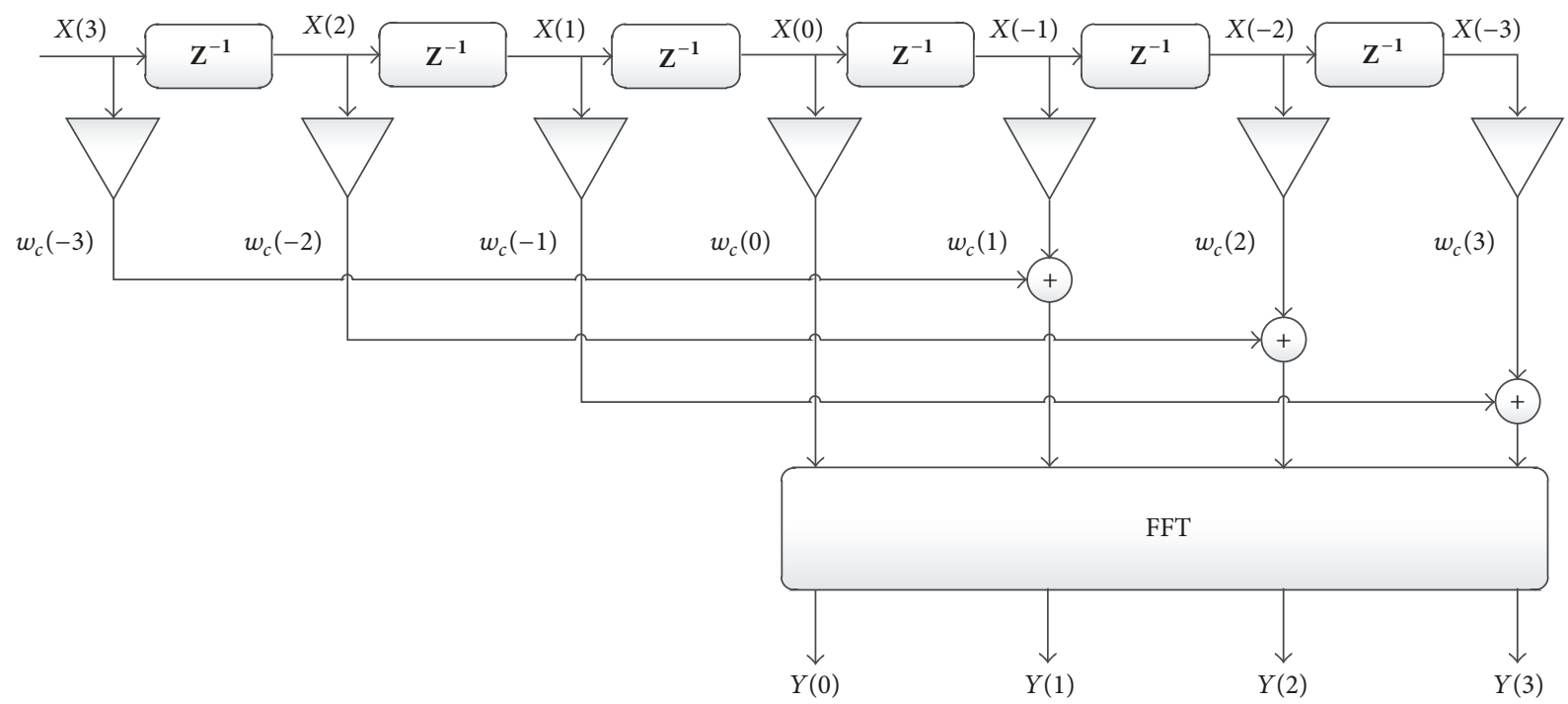

FIGURE 3: AP-FFT spectrum analysis processes.

accuracy. However, the azimuth spectrum broadening will affect the angle resolution. The phase and amplitude error between the velocity channels will affect the DOA estimation. The larger the deviation, the greater the DOA estimation error.

\section{Calibration Methods for Vector Sensor}

Most of the calibration methods of the AVS need the DOAs of the calibrating source. For a single AVS, if the initial phase of is fixed, it can be calibrated by using the trigonometric function relationship between each channel, and the DOA of the source signal can also be estimated. So the sensor can be self-calibrated without knowing the DOAs of the calibrating source in advance.

3.1. AP-FFT Spectrum Analysis Method. In order to achieve high phase estimation precision, the AP-FFT spectrum analysis method is adopted. The AP-FFT algorithm can achieve high phase estimation accuracy and good at antinoise [19$22]$. Its calculated process is shown in Figure 3. Its analysis process is as follows:

(1) A convolution window $\omega_{c}$ is used to weight the data $x(t)$; the length of $\omega_{c}$ is $2 N-1$. The centre of the data is $x(0)$, so the $2 N$ data before and after $x(0)$ are weighted.

(2) After that, add two data whose interval is $N$, then the length of the data is $N$. At last, calculate the FFT to get the frequency spectrum of AP-FFT.

The convolution window $\omega_{c}$ is convolved from the front window $f$ and its reversed window $b$ :

$$
\omega_{c}(n)=f(n) * b(-n), \quad-N+1 \leq n \leq N-1 .
$$

Obviously, if $f(n)$ and $b(n)$ are symmetric, then $\omega_{c}(n)$ become

$$
\omega_{c}(n)=\omega_{c}(-n), \quad-N+1 \leq n \leq N-1 .
$$

If $f(n)=b(n)=R_{N}\left(R_{N}\right.$ is a rectangular window $)$, it is called windowless AP-FFT. If one of $f$ and $b$ is a rectangular window, it is called single window AP-FFT. If $f=b \neq R_{N}$, then it is called two-window AP-FFT. The spectrum $F(j \omega)$ of the causal sequence is

$$
F(j \omega)=F_{g}(j \omega) e^{-j \tau \omega}, \quad \tau=\frac{(N-1)}{2} .
$$

$T$ is the group delay. The FFT of formula (17) is expressed as

$$
W_{c}(j \omega)=F(j \omega) \cdot F^{*}(j \omega)=\left|F_{g}(\omega)\right|^{2} .
$$

As can be seen from the formula above, the spectrum of the convolution window is the square of the front window. By analysing the spectrum $W(j \omega)$, we can get

$$
Y(k)=e^{j \theta_{0}}\left|X_{N}(k)\right|^{2},
$$

where $X_{N}(k)$ is the amplitude spectrum of the FFT and $\theta_{0}$ is the phase of the centre input data, which means that, by extracting $\theta_{0}$, the initial phase of the data can be accurately abstained.

3.2. The Amplitude Estimation Method. According to the previous analysis, if the amplitude and phase are consistent, the velocity channel of the AVS satisfies the following relationship:

$$
a_{x}^{2}+a_{y}^{2}+a_{z}^{2}=a_{P}^{2}
$$

With the existence of nonideal gain responses at each channel, the steering vector $A$ becomes

$$
A^{\prime}=\left[\begin{array}{c}
a_{p}^{\prime} \\
a_{x}^{\prime} \\
a_{y}^{\prime} \\
a_{z}^{\prime}
\end{array}\right]=\left[\begin{array}{c}
g_{p} \cdot a_{p} \\
g_{p} \cdot a_{p} \\
g_{y} \cdot a_{y y} \\
g_{z} \cdot a_{z}
\end{array}\right] .
$$


Apparently, when the gain of each channel is unequal, formula (22) is difficult to satisfy. If sequence of amplitudes is multiplied by a gain correction matrix $\widehat{G}=\operatorname{diag}\left[\widehat{g}_{p}, \widehat{g}_{x}\right.$, $\left.\widehat{g}_{y}, \widehat{g}_{z}\right]$, with $\widehat{G} \cdot G=I$, then

$$
\left(\widehat{g}_{x} \cdot a_{x}^{\prime}\right)^{2}+\left(\widehat{g}_{y} \cdot a_{y}^{\prime}\right)^{2}+\left(\widehat{g}_{z} \cdot a_{z}^{\prime}\right)^{2}=\left(\widehat{g}_{p} \cdot a_{p}^{\prime}\right)^{2} .
$$

Make $\widehat{g}_{p}=1 / A_{p}$, and define

$$
\begin{gathered}
\widetilde{A}=\frac{\left[a_{x}^{\prime 2}, a_{y}^{\prime 2}, a_{y}^{\prime 2}\right]^{T}}{a_{p}^{\prime 2}}, \\
\widetilde{G}^{\prime}=\left[\hat{g}_{x}^{2}, \widehat{g}_{y}^{2}, \widehat{g}_{z}^{2}\right]^{T} .
\end{gathered}
$$

It is obvious that $\widetilde{A} \cdot \widetilde{G}^{\prime}=1$. If there are several sets of measurements in different angles, define

$$
W=\left[\widetilde{A}_{1}, \widetilde{A}_{2}, \ldots, \widetilde{A}_{n}\right]^{T} .
$$

The maximum rank of $W$ is 3 ; it will be positive. Then the least square estimation method can be used to evaluate $\widehat{G}$ :

$$
\begin{aligned}
\widetilde{G}^{\prime} & =\left(W^{T} W\right)^{-1} W^{T} \cdot H, \\
G & =\frac{1}{\sqrt{\widetilde{G}^{\prime}}} .
\end{aligned}
$$

So the estimation of the amplitude gain matrix is obtained by using the above formula.

3.3. The Proposed Algorithm. According to the previous analysis, the initial phase of each channel can be achieved by using the AP-FFT algorithm. For the calibration of AVS, the relative phase difference between each channel is more concerned. The amplitude calibration has the same needs. In this paper, the phase and amplitude of the sound pressure channel are taken as the reference quantity. And then the self-calibration algorithm based on AP-FFT is proposed. The specific steps are as follows:

(1) Calculate AP-FFT to the measurement $S_{1}$ of each channel, and then extract the phase $\phi_{1}=\left[\varphi_{p 1}, \varphi_{x 1}\right.$, $\left.\varphi_{y 1}, \varphi_{z 1}\right]^{T}$ and amplitude $\widehat{A}_{1}=\left[\widehat{a}_{p 1}, \widehat{a}_{x 1}, \widehat{a}_{y 1}, \widehat{a}_{z 1}\right]^{T}$ at the central frequency $f_{0}$ of the spectrum.

(2) Acquire the phase difference estimation $\Delta \varphi_{1}=$ $\left[\Delta \varphi_{p x}, \Delta \varphi_{p y}, \Delta \varphi_{p z}\right]^{T}$ between each vibration velocity channel and the sound pressure channel. Compute the square of the each channel's amplitude $\widehat{A}_{1}$; the result is defined as $\widehat{A}_{1}^{2}$. The amplitude of each vibration velocity channel is divided by the pressure channel. Then get the vector $\widetilde{A_{1}}$.

(3) Get the measurement $S_{2}, S_{3}, \ldots, S_{m}, m>3$ from different incident angles. Then repeat steps (1) and (2) to get $\Delta \phi_{m}$ and $\widetilde{A}_{m}$.
(4) Average all the phase difference estimation $\phi_{i}(i=$ $1, \ldots, m)$ to obtain the phase difference estimation. Use the amplitude estimation vector $\widetilde{A}_{i}(i=1, \ldots, m)$ to compose the amplitude gain matrix $W$. Estimate the amplitude gain by using formula (27).

(5) Modify the sampling data by the phase and gain estimation result. Then calculate the DOA of the incident signal.

The framework of the proposed algorithm is shown in Figure 4. In practical application, we use the two-dimension vector to estimate the horizontal angle of the DOA more than the three-dimension AVS. In this situation, two sets of measurements are enough to calibrate the vector sensor and estimate the bearing.

\section{Simulation Analyses}

Simulation experiments are carried out to verify the method proposed in this paper. As the AVSs are usually used to estimate the horizontal direction, the simulation also selected two-dimension AVS for analysing. Without losing generality, the amplitude gain factor of $x$ and $y$ channels is randomly distributed between $(0.6,1.4)$, and the phase is randomly distributed between $(-90,90)^{\circ}$.

Firstly, the accuracy of the phase difference estimation is analysed. The length of $S_{1}$ is 512. 500-time Monte Carlo simulation is executed under different SNR (signal-to-noise ratio), using the ME (mean error) and the RMSE (root mean square error) to show the validity of this algorithm. These two parameters are calculated as

$$
\begin{aligned}
\text { Mean } & =\frac{1}{M} \sum_{m=1}^{M}\left|\widetilde{\varphi}_{m}-\varphi_{m}\right|, \\
\text { RMSE } & =\sqrt{\frac{1}{M} \sum_{m=1}^{M}\left(\widetilde{\varphi}_{m}-\varphi_{m}\right)^{2},}
\end{aligned}
$$

where $\|$ means calculating the absolute value, $M$ is the number of Monte Carlo simulation, and $\widetilde{\varphi}_{m}$ and $\varphi_{m}$ are the estimation and true value of the phase. The simulation result is shown in Figure 5.

It can be seen from the figure that the estimation accuracy of the AP-FFT algorithm is improved following the increase of SNR. When SNR $=-10 \mathrm{~dB}$, the phase estimation error is still less than $7^{\circ}$; when SNR $=-5 \mathrm{~dB}$, the ME and RMSE are all less than $2^{\circ}$; in this situation, the influence of the phase error to the DOA estimation is very small and can be neglected. Change the AP-FFT number to 1024; other conditions remain the same; the simulation results are shown in Figure 6. It is obvious that, as the increasing of the calculation points, the estimation accuracy is improved; when $\mathrm{SNR}=0 \mathrm{~dB}$, the ME and RMSE are all less than $2^{\circ}$. It means that as the SNR is low, we can improve the estimation accuracy by increasing the number of the AP-FFT.

Secondly, the validity of amplitude gain estimation is verified. Assume that there are two sets of measurement and only one target, the DOA of the incident signal are 


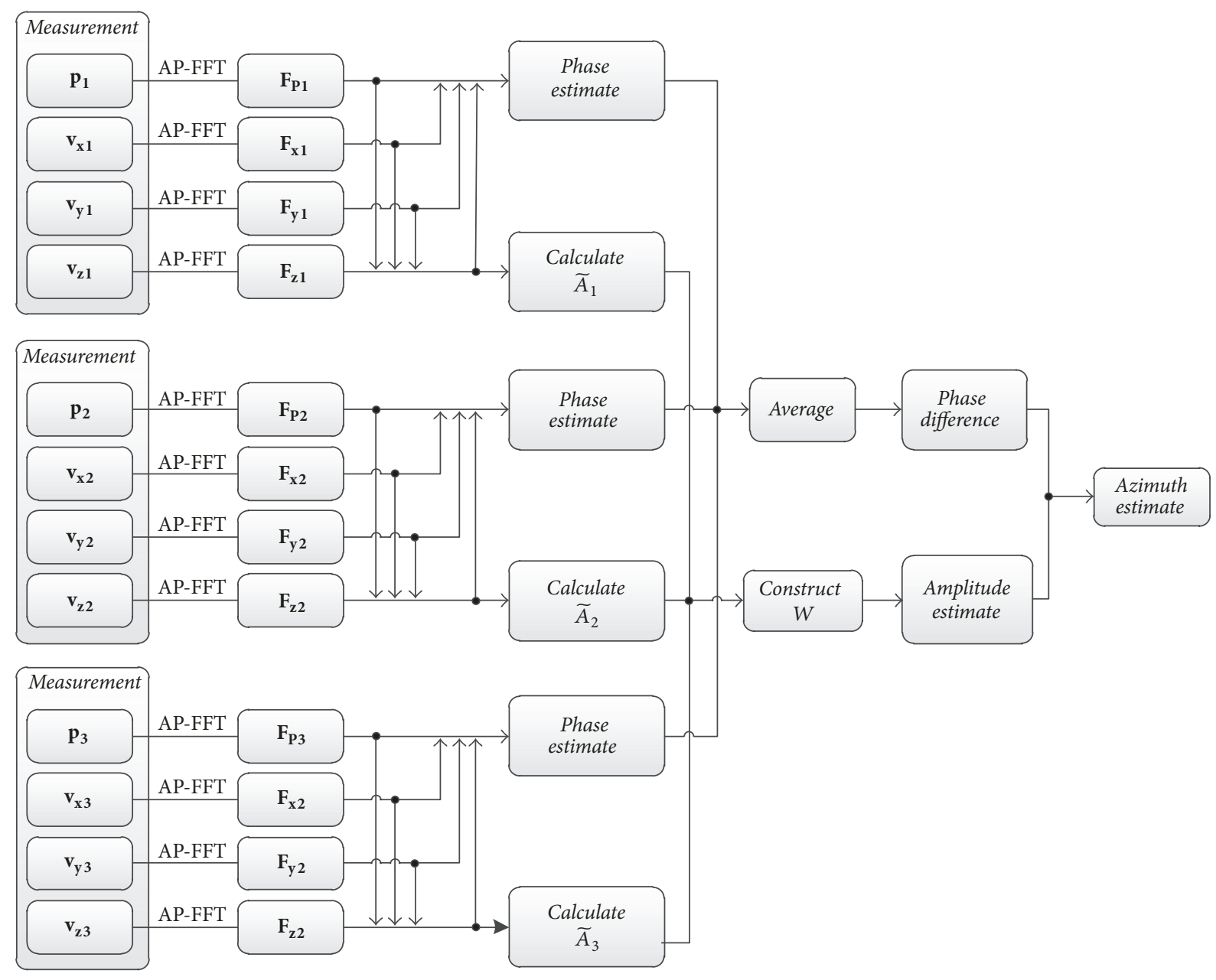

FIgURE 4: Framework of the proposed algorithm.

$25^{\circ}$ and $70^{\circ}$, and the number of AP-FFT is 1024 . The amplitude estimation RMSE with different SNR is shown in Figure 7.

As the simulation result shows, the amplitude estimation accuracy of the proposed algorithm is higher when SNR is improved. When the SNR is $-10 \mathrm{~dB}$, the estimation result is poor. When the SNR is $0 \mathrm{~dB}$, the RMSE result is close to 0.05 . The result of the two velocity channel shows little difference in the simulation. This is due to the different input SNR of the two channels, when the incident signal comes from different directions, the steering vector of the velocity channel changes that makes the input SNR of each velocity channel different. When the input SNR is raised, the two simulation results tend to be the same. In order to ensure the accuracy of estimation, the angel of the target should avoid appearing in the smaller range of the steering vector, which means the angle should be as far away as possible from $90^{\circ}$ and its integer times' angle.

The amplitude influence of the angle difference between two DOA angles is then analysed. The bearing of the target 1 is fixed at $45^{\circ}$, the bearing of target 2 changed from 0 to $90^{\circ}$, and the rest of the conditions keep the same as prior simulation. The amplitude estimation ME curve via SNR is shown in Figure 8 (channels $x$ and $y$ have similar simulation result, so only the result of the channel $x$ is listed here).

It is obvious that the amplitude estimation accuracy is related to the difference of the incident angle. In the same SNR, if the angle difference is small, the estimation result is bad. In order to obtain high estimation accuracy, the difference of the incident angle should be big; as the increasing of the SNR, the angle difference requirement is also getting lower and lower. When the SNR is $5 \mathrm{~dB}$ and the angle difference is greater than $10^{\circ}$, the estimation result could be high-accuracy.

At last, the effectiveness of the algorithm is verified. Without losing generality, the DOA of the incident signal is distribution in $(-180,180)^{\circ}$. In order to reduce the influence of the angle difference, the difference of the two incident angles is set to be larger than $20^{\circ}$ and makes them $10^{\circ}$ away from the external value of the trigonometric function. After estimating the phase and amplitude, the result is used to calibrate the measurement and compute the DOA. Compare the angles estimation result with the result without modification. The results are shown in Figure 8 (because the two angle estimation results are nearly the same, only one comparison result is plotted in this diagram draw). 


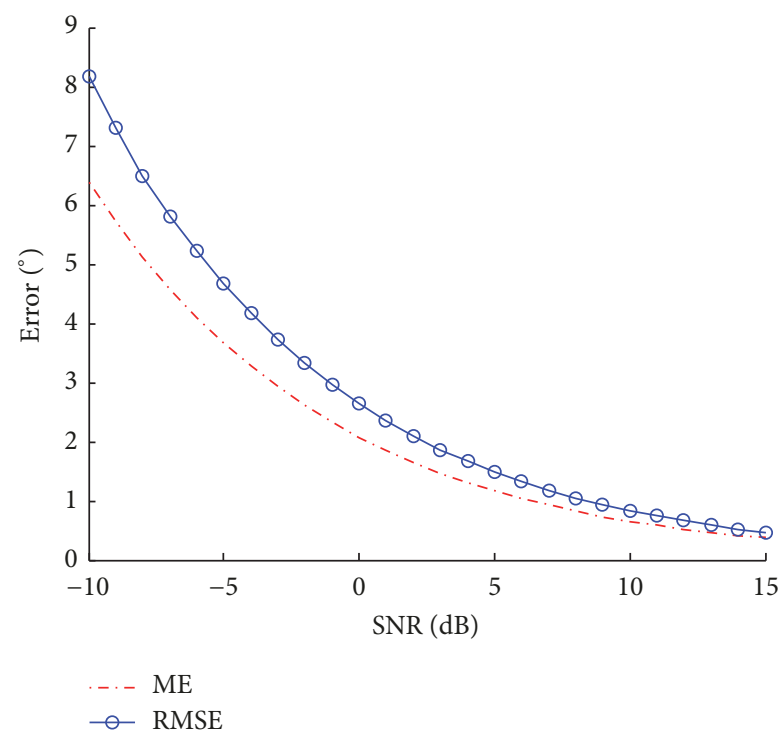

FIGURE 5: Phase estimation error curve via SNR.

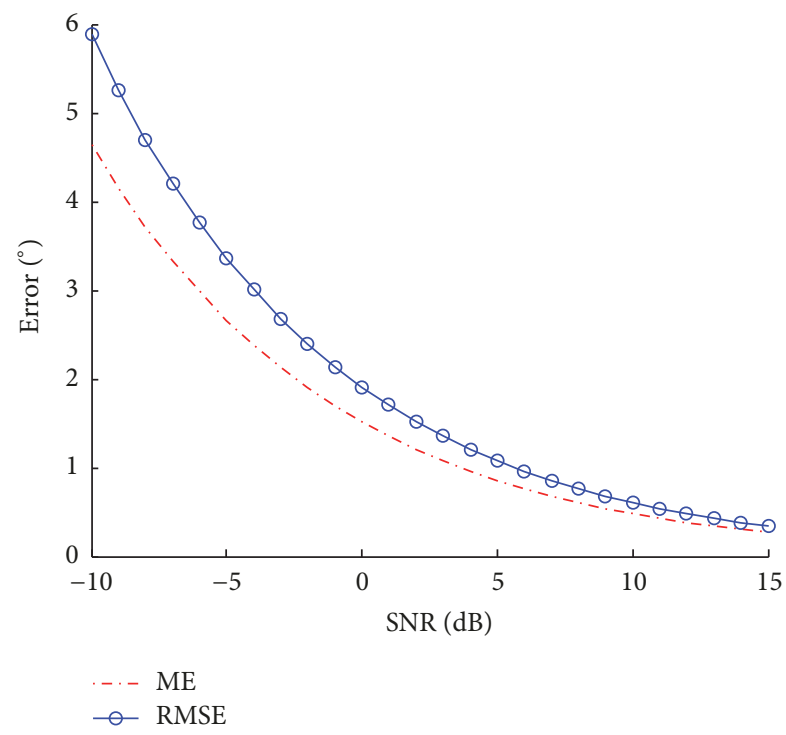

FIGURE 6: Phase estimation error curve via SNR.

As can be seen from Figure 9, there is a significant deviation from the estimation of the DOA in the presence of amplitude and phase errors, and it is independent of SNR. With the algorithm proposed in this paper, the amplitude and phase error of each channel are corrected effectively. The target azimuth estimation accuracy is improved, and with the increase of the SNR, the estimation accuracy also increases. When the SNR is $0 \mathrm{~dB}$, the ME and RMSE are all less than $4^{\circ}$; when the SNR is $10 \mathrm{~dB}$, the estimation results have been less than $1.5^{\circ}$; when the SNR is $20 \mathrm{~dB}$, all the estimation results are less than $0.5^{\circ}$.

\section{Conclusions}

Aiming at the problem of amplitude and phase deviation of the AVS, the self-calibration technique is studied in this

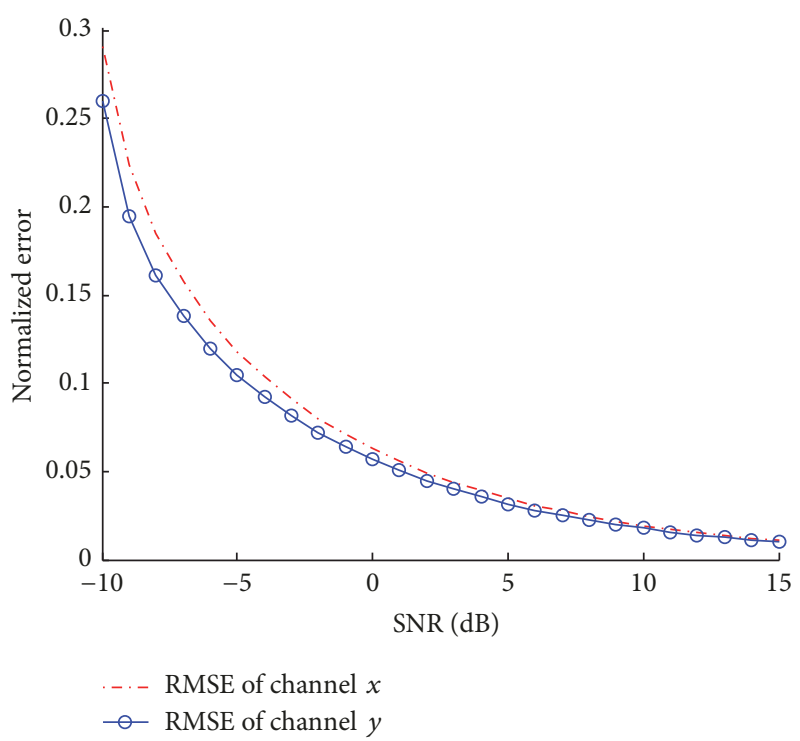

FIGURE 7: Gain estimation error curve via SNR.

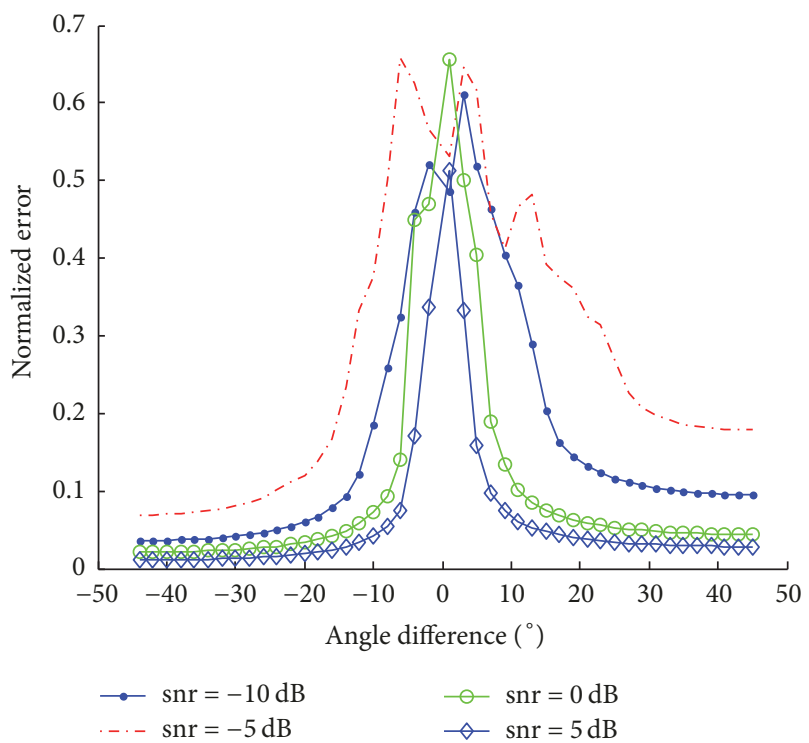

FIGURE 8: Gain estimation error curve via different angle.

paper. Different from other aided methods, this method can fix the phase and amplitude uncertainties without prior knowledge of the DOAs of the calibrating source. Using the AP-FFT, high precision phase estimation can be obtained. And concerding the relationship of different velocity channels, a self-calibration algorithm based on APFFT is proposed. The initial phase estimation is derived by calculating the AP-FFT to each channel. By using the result above and measurement from different incident angles that obey certain relationship, we can construct a matrix to estimate the amplitude gain of each channel. Then the high-accuracy DOA of incident signal can be obtained. 


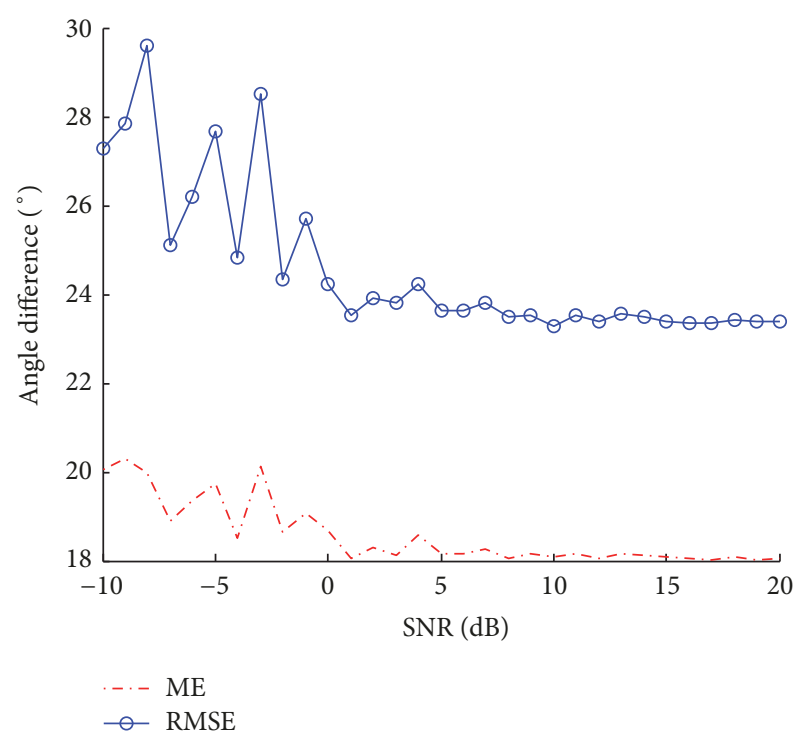

(a) The results without calibration

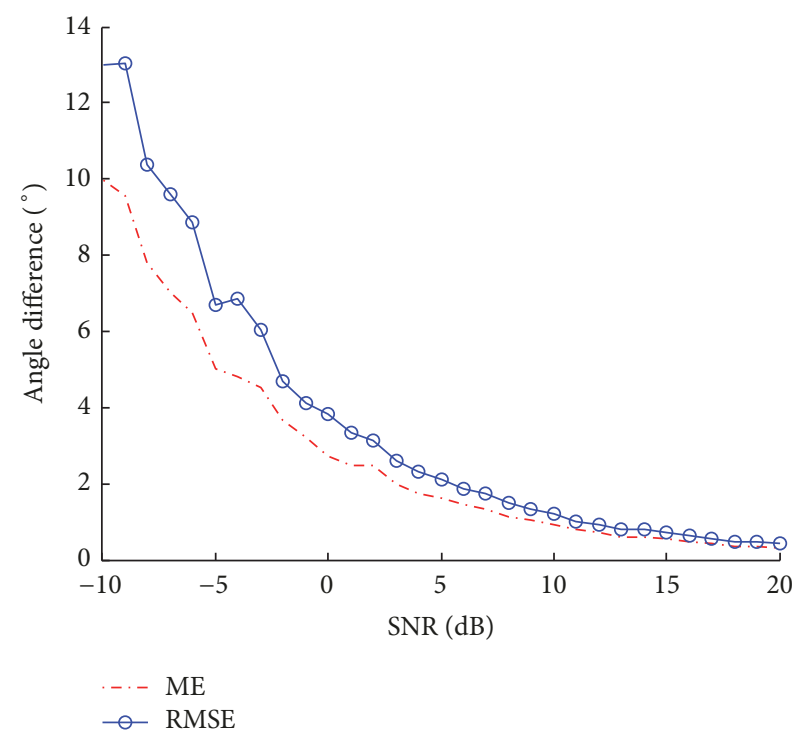

(b) The results with calibration

Figure 9: Angle estimation errors via SNR.

\section{Conflicts of Interest}

The authors declare that there are no conflicts of interest regarding the publication of this paper.

\section{Acknowledgments}

This work was supported by State Key Laboratory of Robotics Foundation (nos. 2016-Z08 and 2017-Z05), the Basic Research Project of Technology (no. JSJL2016604B003), the Heilongjiang Postdoctoral Research Startup Fund (no. LBHQ15025), and the Open Fund of Qingdao National Laboratory for Marine Science and Technology (no. QNLM2016-0RP0 102).

\section{References}

[1] A. Abdi and H. Guo, "Signal correlation modeling in acoustic vector sensor arrays," IEEE Transactions on Signal Processing, vol. 57, no. 3, pp. 892-903, 2009.

[2] J. Cao, J. Liu, J. Wang, and X. Lai, "Acoustic vector sensor: Reviews and future perspectives," IET Journals on The Institution of Engineering and Technology, vol. 11, no. 1, pp. 1-9, 2017.

[3] P. Tichavský, K. T. Wong, and M. D. Zoltowski, "Near-field/farfield azimuth and elevation angle estimation using a single vector hydrophone," IEEE Transactions on Signal Processing, vol. 49, no. 11, pp. 2498-2510, 2001.

[4] A. Nehorai and E. Paldi, "Acoustic vector-sensor array processing," IEEE Transactions on Signal Processing, vol. 42, no. 9, pp. 2481-2491, 1994.

[5] Y. Song and K. T. Wong, "Three dimensional localization of a near-field emitter of unknown spectrum using an acoustic vector sensor," IEEE Transactions on Aerospace and Electronic Systems, vol. 49, no. 2, pp. 1035-1041, 2013.

[6] Y. I. Wu, S.-K. Lau, and K. T. Wong, "Near-field/far-field array manifold of an acoustic vector-sensor near a reflecting boundary," The Journal of the Acoustical Society of America, vol. 139, no. 6, pp. 3159-3176, 2016.

[7] X. Zhong, A. B. Premkumar, and H. Wang, "Multiple wideband acoustic source tracking in 3-D space using a distributed acoustic vector sensor array," IEEE Sensors Journal, vol. 14, no. 8, pp. 2502-2513, 2014.

[8] Y. I. Wu and K. T. Wong, "Acoustic near-field sourcelocalization by two passive anchor-nodes," IEEE Transactions on Aerospace and Electronic Systems, vol. 48, no. 1, pp. 159-169, 2012.

[9] Y. I. Wu, K. T. Wong, and S.-K. Lau, "The acoustic vectorsensor's near-field array-manifold," IEEE Transactions on Signal Processing, vol. 58, no. 7, pp. 3946-3951, 2010.

[10] P. K. Tam and K. T. Wong, "Cramer-Rao bounds for direction finding by an acoustic vector-sensor under non-ideal gainphase responses, non-collocation, or non-orthogonal orientation," IEEE Sensors Journal, vol. 9, no. 8, pp. 969-982, 2009.

[11] B. Xu, J. Wind, H.-E. De Bree, T. Basten, and E. Druyvesteyn, "Amplitude, phase, location and orientation calibration of an acoustic vector sensor array, part I: Theory," Journal of the Acoustical Society of America, vol. 127, no. 3, pp. 1-11, 2010.

[12] M. Pesavento, A. B. Gershman, and K. M. Wong, "Direction finding in partly calibrated sensor arrays composed of multiple subarrays," IEEE Transactions on Signal Processing, vol. 50, no. 9, pp. 2103-2115, 2002.

[13] Y. Song, K. T. Wong, and F. Chen, "Quasi-blind calibration of an array of acoustic vector-sensors that are subject to gain errors/mis-location/mis-orientation," IEEE Transactions on Signal Processing, vol. 62, no. 9, pp. 2330-2344, 2014.

[14] C. M. S. See, "Method for array calibration in high-resolution sensor array processing," IEE Proceedings Radar, Sonar and Navigation, vol. 142, no. 3, pp. 90-96, 2002.

[15] A. Contini, A. Canclini, F. Antonacci, M. Compagnoni, A. Sarti, and S. Tubaro, "Self-calibration of microphone arrays from measurement of times of arrival of acoustic signals," in Proceedings of the 5th International Symposium on Communications 
Control and Signal Processing, ISCCSP 2012, 6, 1 pages, Rome, Italy, May 2012.

[16] H. S. Lim, K. Rameshchandra, and S. T. Wei, "Estimation and compensation of rotation perturbation in linear $2 \mathrm{~d}$ acoustic vector sensor array," in Proceedings of IEEE Conference on OCEANS 2011, pp. 1-8, Santander, Spain, June 2011.

[17] X. Yuan, "Direction-finding with a misoriented acoustic vector sensor," IEEE Transactions on Aerospace and Electronic Systems, vol. 48, no. 2, pp. 1809-1815, 2012.

[18] Y. Song, "Blind calibration and remedy for mis-oriented velocity-sensor triads," in Proceedings of the 2013 38th IEEE International Conference on Acoustics, Speech, and Signal Processing, ICASSP 2013, pp. 3880-3884, Vancouver, BC, Canada, May 2013.

[19] D. Billon and F. Fohanno, "Theoretical performance and experimental results for synthetic aperture sonar self-calibration," in Proceedings of IEEE Conference on OCEANS 1998, pp. 965-970, Nice, France, August 1998.

[20] F. Jinlin, H. Chunping, Y. Lei, and L. Ke, "All phase-FFT signal detection method for QAM system in fast-moving wireless channel," in Proceedings of the 2009 4th IEEE Conference on Industrial Electronics and Applications, ICIEA 2009, pp. 759762, Xi'an, China, June 2009.

[21] L. Huai, G. E. Sobelman, and X. Zhou, "Wideband spectrum sensing using the all-phase FFT," in Proceedings of the 2011 IEEE 9th International Conference on ASIC, ASICON 2011, pp. 208211, Xiamen, China, October 2011.

[22] X. Lu and Y. Zhang, "Phase detection algorithm and precision analysis based on all phase FFT," in Proceedings of the International Conference on Automatic Control and Artificial Intelligence, ACAI 2012, pp. 1564-1567, Xiamen, China, March 2012. 


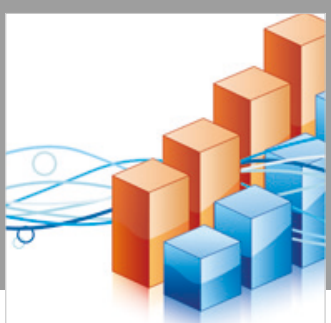

Advances in

Operations Research

\section{-n-m}
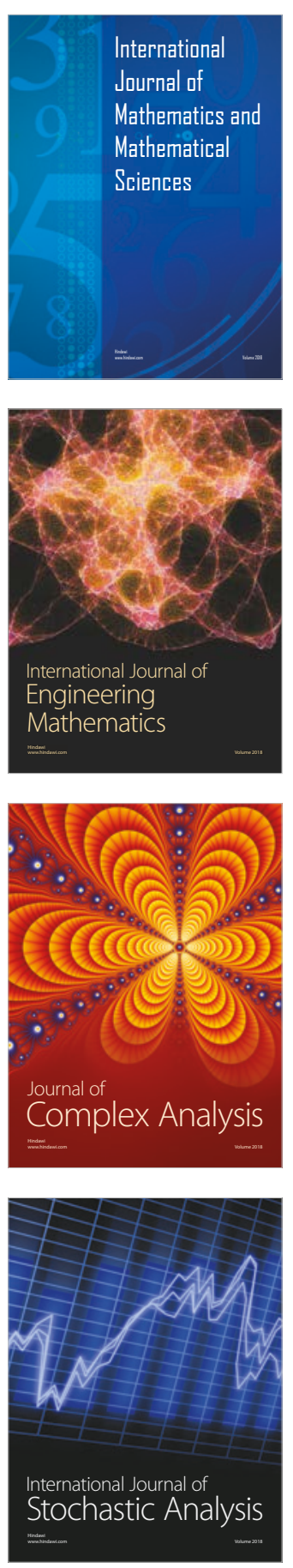
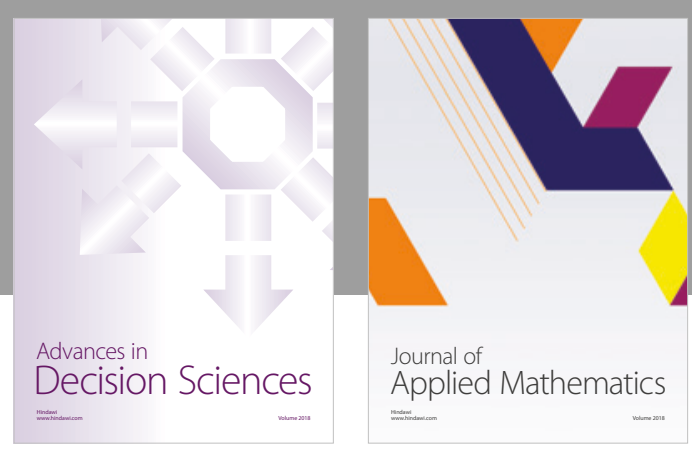

Journal of

Applied Mathematics
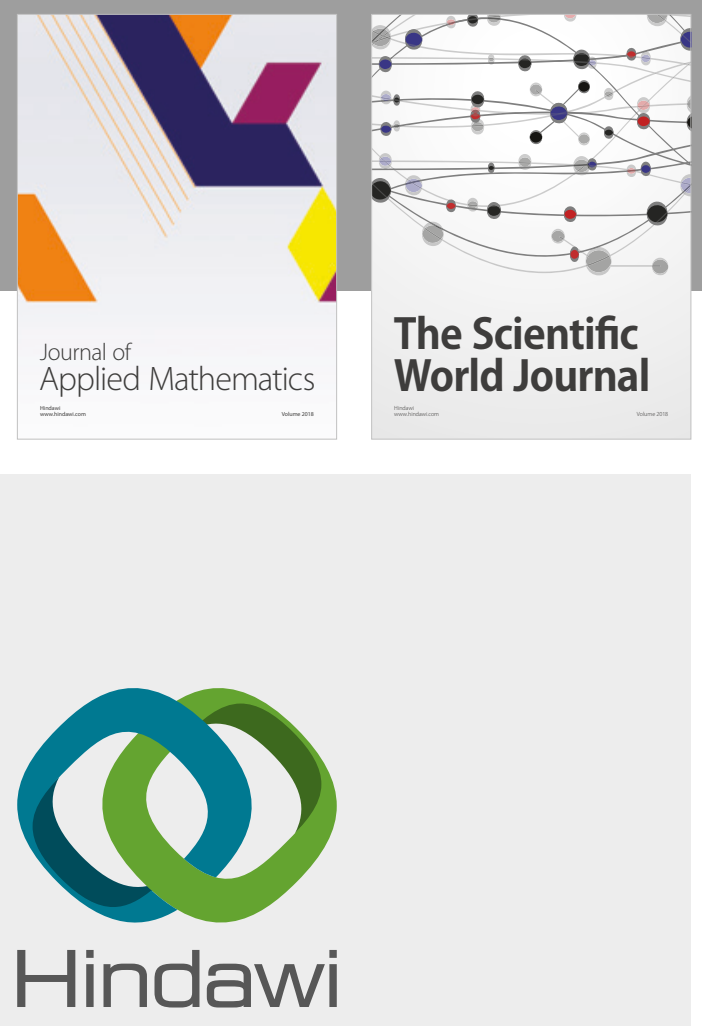

Submit your manuscripts at

www.hindawi.com

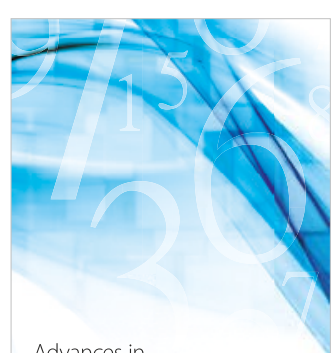

Advances in
Numerical Analysis
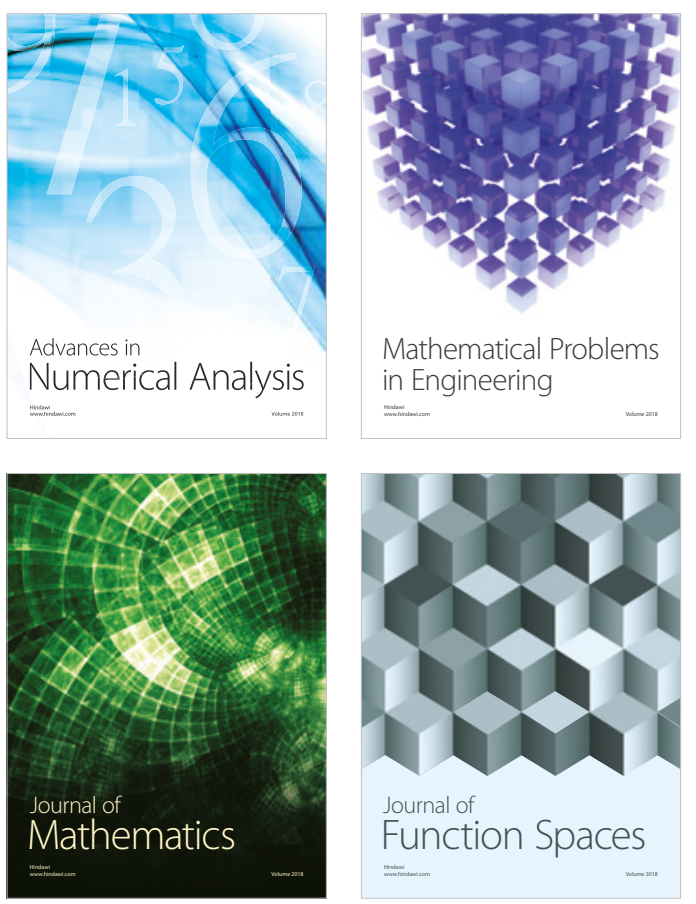

Mathematical Problems in Engineering

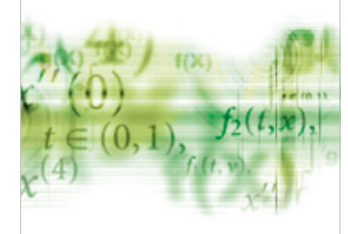

International Journal of

Differential Equations

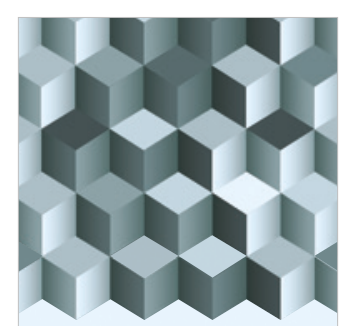

Journal of

Function Spaces

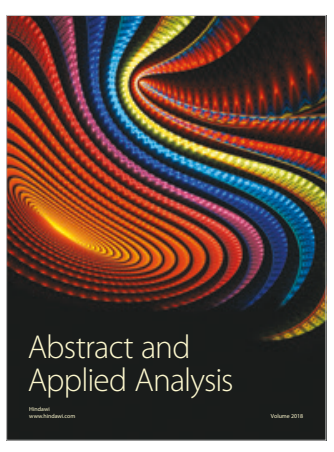

The Scientific

World Journal

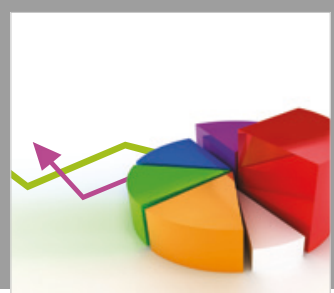

Journal of

Probability and Statistics
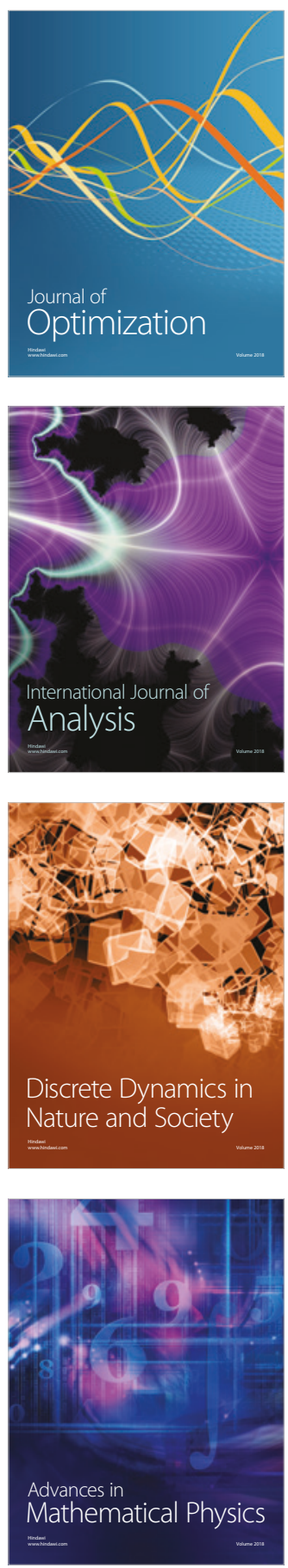